\title{
GEM on proliferation and apoptosis of childhood AL cells through inhibiting c-myc expression by upregulating miR-125a-3p
}

\author{
WEIWEI XING $^{1 *}$, YUXIA YIN $^{2 *}$, SAINA YANG $^{2}$ and GUANG LU ${ }^{3}$ \\ Departments of ${ }^{1}$ Pediatrics, ${ }^{2}$ Neurosurgery Ward II, Yidu Central Hospital of Weifang, Weifang, Shandong 262500; \\ ${ }^{3}$ Department of Hematology, Shengli Oilfield Central Hospital, Dongying, Shandong 257034, P.R. China
}

Received October 18, 2019; Accepted December 17, 2019

DOI: $10.3892 / \mathrm{ol} .2020 .11396$

\begin{abstract}
Effect of gemcitabine (GEM) on proliferation and apoptosis of childhood acute leukemia (AL) cells and the mechanism of action were investigated. Bone marrow and peripheral blood of 18 newly diagnosed children with childhood AL admitted to Yidu Central Hospital of Weifang were selected, and the miR-125a-3p level in peripheral blood of healthy children and children with AL was detected by quantitative reverse transcription-polymerase chain reaction (qRT-PCR). Leukemia cells from the bone marrow of children with AL were primarily cultured and purified to observe the morphology. miR-125a-3p mimic was transfected into childhood AL cells. The cells were randomly divided into three groups: control group, GEM group and GEM + miR-125a-3p mimic group. 5-ethynyl-2'-deoxyuridine (EdU) staining assay was chosen to detect the proliferation of childhood AL cells in each group. Terminal deoxynucleotidyl transferase dUTP nick end labeling (TUNEL) staining assay was adopted to determine apoptosis of childhood AL cells. The protein level of c-myc was measured via western blotting. Compared with that in the healthy children, the level of miR-125a-3p in the peripheral blood of children with AL was remarkably decreased. Compared with those in the control group, GEM inhibited proliferation and promoted apoptosis of childhood AL cells, and impeded the protein expression of c-myc in these cells. Compared with those in the GEM group, GEM + miR-125a-3p mimic notably reduced the proliferation and enhanced apoptosis of cells, and the protein expression of c-myc in cells was overtly reduced. The level of miR-125a-3p in peripheral blood of children with AL is obviously decreased. It is suggested in
\end{abstract}

Correspondence to: Dr Guang Lu, Department of Hematology, Shengli Oilfield Central Hospital, 31 Jinan Road, Dongying, Shandong 257034, P.R. China

E-mail: yunc695@163.com

*Contributed equally

Key words: microRNA, childhood AL, proliferation, apoptosis, c-myc this study that GEM can inhibit the proliferation and promote apoptosis of childhood AL cells, and the mechanism may be related to upregulated miR-125a-3p inhibiting the expression of c-myc.

\section{Introduction}

Childhood acute leukemia (AL) is the most common malignant tumor that invades the blood system of children. It is also the malignant tumor of children with the highest incidence rate, which seriously threatens the life and health of children (1-3). At present, the pathogenesis of childhood AL remains unclear. It is generally believed that its occurrence is closely related to genetic and environmental factors, but there are still no measures to prevent and control the occurrence of this disease (4). Therefore, it is necessary for people to further understand and explore the pathogenesis of childhood AL, which is of great significance for its prevention and treatment in the future.

As a novel deoxycytidine analog and nucleoside reductase inhibitor, gemcitabine (GEM) is a metabolic anticancer drug with a molecular weight of 263.20 and a molecular formula of $\mathrm{C}_{9} \mathrm{H}_{11} \mathrm{~F}_{2} \mathrm{~N}_{3} \mathrm{O}_{4}$ (5). Clinically, it is mainly applied as a second-line drug for advanced pancreatic cancer adopted when fluorouracil treatment fails, which can markedly improve patients' quality of life. Besides, this drug exhibits a highly curative effect on metastatic non-small cell lung cancer. Studies have denoted that GEM can act on the DNA synthesis stage in tumor cells to inhibit DNA synthesis and make DNA strand breaks. In addition, a study has pointed out that GEM can also impede the self-repair function of DNA (6). It is worth noting that the side effects of the drug become more serious as the infusion time prolongs. Especially for elderly patients with poor renal function, appropriate dose reduction or intermittent administration should be adopted in clinical treatment (7). A large number of studies have confirmed that GEM performs well in treating non-small cell lung cancer and pancreatic cancer, but there are few reports on whether GEM can be used to treat childhood AL.

Micro ribonucleic acids (miRNAs) are a kind of non-coding single-stranded RNAs with a length of 20-24 nucleotides, which participate in the biological processes of regulating 
individual development, growth, and proliferation, differentiation and apoptosis of cells (8-10). Some studies have pointed out that over $90 \%$ of genes are regulated by miRNAs. Thus, it is essential to investigate the regulatory effect of miRNAs in physiological and pathological processes of the body. Some studies have discovered that the level of miR-125a-3p declines in the case of acute myeloid leukemia, but there are few reports on whether GEM can affect the proliferation and apoptosis of childhood AL cells by regulating the level of miR-125a-3p.

In this study, the level of miR-125a-3p in the peripheral blood of healthy children and AL children was compared. The results revealed that the level of miR-125a-3p in the peripheral blood of AL children was overtly reduced. Then, childhood AL cells were primarily cultured and purified. After incubation, the effect of GEM on proliferation and apoptosis of childhood AL cells was observed and its mechanism of action is reported.

\section{Materials and methods}

Reagents. GEM was from Jianze Co., 1640 medium and fetal bovine serum were from HyClone, culture plates and bottles were from Corning, the 5-ethynyl-2'-deoxyuridine (EdU) and terminal deoxynucleotidyl transferase dUTP nick end labeling (TUNEL) kits were from Nanjing KeyGen Biotech Co., Ltd., miR-125a-3p mimic and miRNA extraction kit were from Shanghai Gene Pharma Co., Ltd., MTT was from Amresco, LLC, SYBR Primer Ex Taq fluorescent quantitative polymerase chain reaction (PCR) kit was from Takara, miR-125a-3p and U6 primers were from Applied Biosystems; Thermo Fisher Scientific, Inc., and c-myc, reduced glyceraldehyde-phosphate dehydrogenase (GAPDH) primary anti-antibody and hypothalamic regulatory peptides (HRP) labeled secondary antibody were from Beijing Bioss Biotechnology Co., Ltd.

Instruments. The fluorescent inverted microscope was from Olympus. The $\mathrm{CO}_{2}$ incubator was from Thermo Fisher Scientific, Inc. The fluorescence quantitative PCR instrument was from Applied Biosystems; Thermo Fisher Scientific, Inc. The ultrapure water meter was from Millipore, France. The high-speed low-temperature centrifuge was from Beckman Coulter, Inc.

Clinical data. Nineteen untreated children (10 males and 9 females) with AL diagnosed by bone marrow cell morphology analysis, flow cytometry immunotyping and fusion gene in Yidu Central Hospital of Weifang (Weifang, China) from August 2015 to September 2018 were selected. Nine children with normal bone marrow and without hematological diseases were randomly selected as control. This study was conducted with the informed consent of family members of the children. The study was approved by the Ethics Committee of Yidu Central Hospital of Weifang.

\section{Methods}

Detection of miR-125a-3p level in peripheral blood of healthy children and children with $A L$ by quantitative reverse transcription-PCR ( $q R T-P C R)$. The peripheral blood of children in each group was collected and added with TRIzol reagent. Total RNA in each group of blood was extracted and reverse transcribed into complementary deoxyribonucleic acid (cDNA),
Table I. Sequence information of miR-125a-3p and U6 primers.

\begin{tabular}{ll}
\hline Gene name & \multicolumn{1}{c}{ Primer sequences } \\
\hline miR-125a-3p & $\begin{array}{l}\text { ACGTGTGTAGCTTATCAGACTG } \\
\text { AATGGTTGTTCTCCACACTCTC } \\
\text { U6 }\end{array}$ \\
& GCGCGTCGTGAAGCGTTC \\
& GTGCAGGGTCCGAGGT
\end{tabular}

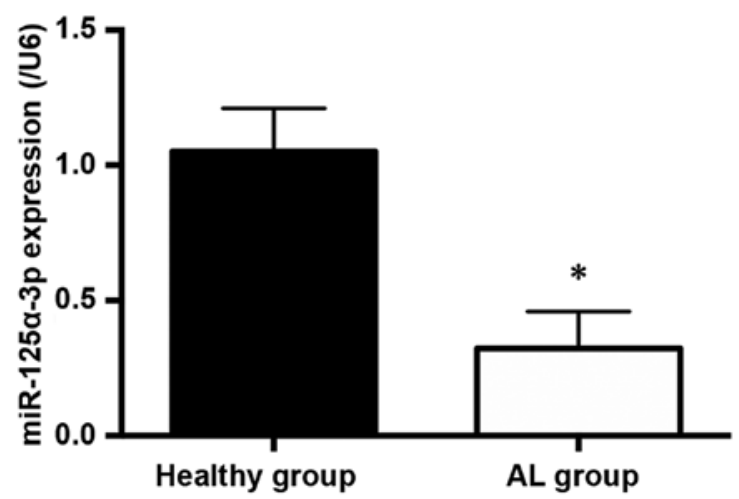

Figure 1. Expression of miR-125a-3p. "P $<0.05$, AL children group vs. healthy children group). AL, acute leukemia.

and the target gene fragment was amplified adopting the first strand cDNA as a template. The sample was put into a fluorescence quantitative PCR instrument. Information on the primers is provided in Table I with U6 gene as the internal reference.

Culture and transfection of childhood AL cells. Under aseptic conditions, the bone marrow samples collected were added with separation liquid, centrifuged at $200 \mathrm{x} \mathrm{g}$ for $15 \mathrm{~min}$ at $4^{\circ} \mathrm{C}$, and then the intermediate cell layer was inhaled and transferred into a new EP tube, washed with phosphate-buffered saline (PBS) solution and centrifuged again at $200 \mathrm{x} g$ for $5 \mathrm{~min}$ at $4^{\circ} \mathrm{C}$. The supernatant was discarded, and the suspended sediment was inoculated in a culture bottle with 1640 complete culture medium, placed in a $\mathrm{CO}_{2}$ incubator to start the culture, and subcultured after 3-5 days. The cells were randomly divided into three groups: GEM group $(10 \mu \mathrm{g} / \mathrm{ml}$, $24 \mathrm{~h}$ ), GEM + miR-125a-3p mimic group (when the cell fusion degree was over $80 \%$, miR-125a-3p mimic was transfected into childhood AL cells using Lipofectamine 2000 transfection technology, with GEM final concentration reaching $10 \mu \mathrm{g} / \mathrm{ml}$ ), and control group (childhood AL cells without any treatment).

Detection of GEM's effect on proliferation of childhood AL cells through EdU staining. Cells in each group were fixed with $4 \%$ paraformaldehyde for $15 \mathrm{~min}$, and permeabilized with $0.3 \%$ Triton X-100 solution for $15 \mathrm{~min}$. EdU staining solution was added to each well and protected from light for $30 \mathrm{~min}$. After washing with PBS solution, the anti-fluorescence quencher was added, and staining was observed under a fluorescence microscope.

Detection of the GEM effect on apoptosis of childhood AL cells by TUNEL staining. Cells in each group were collected, fixed 

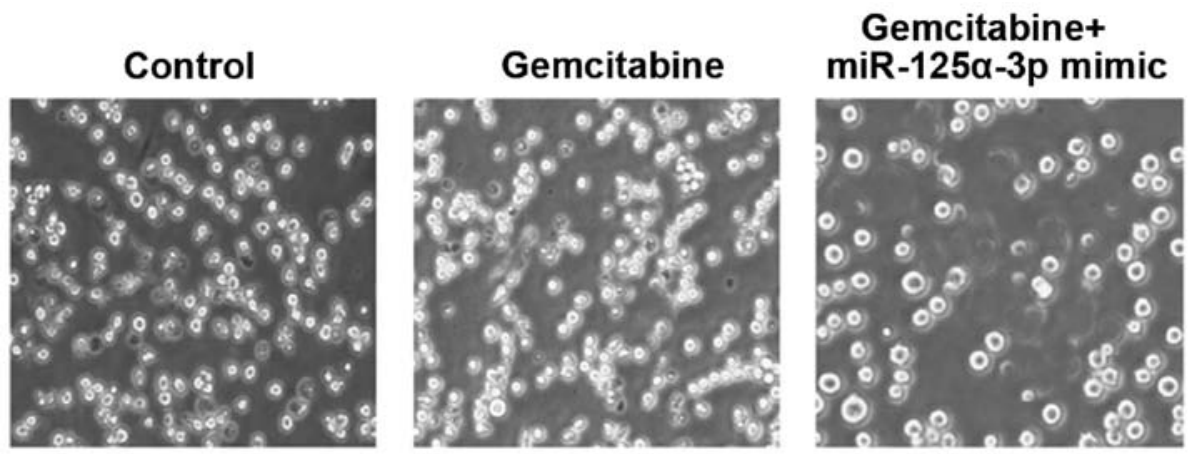

Figure 2. Childhood AL cell morphology observed under a light microscope (x20). AL, acute leukemia.

A

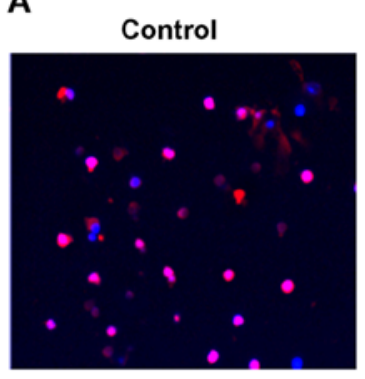

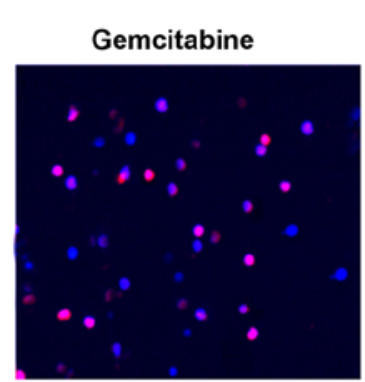

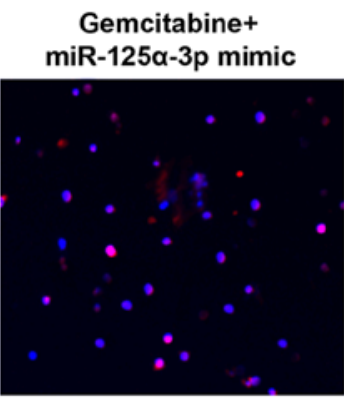

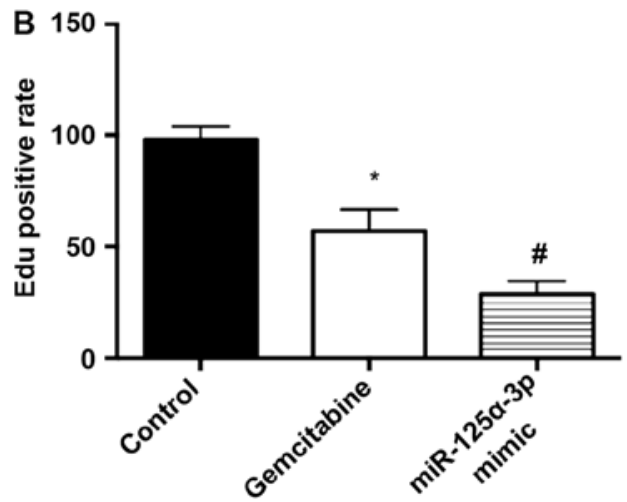

Figure 3. Cell proliferation in each group detected via EdU assay. (A) EdU staining (x20). (B) Proliferation rate. *P<0.05, GEM group vs. control group; ${ }^{\#} \mathrm{P}<0.05, \mathrm{GEM}+\mathrm{miR}-125 \mathrm{a}-3 \mathrm{p}$ mimic group vs. GEM group. EdU, 5-ethynyl-2'-deoxyuridine; GEM, gemcitabine.

and permeabilized as indicated above. Each well of cells was added with $50 \mu \mathrm{l}$ of TUNEL detection solution, then incubated at $37^{\circ} \mathrm{C}$ in the dark for $60 \mathrm{~min}$, washed three times with PBS solution for $5 \mathrm{~min}$, added with anti-fluorescence quencher, and finally placed under a microscope to observe the TUNEL staining.

Detection of c-myc protein level in childhood AL cells via western blotting. Each group of cells were lysed utilizing RIPA lysate, centrifuged at $500 \mathrm{xg}$ for $5 \mathrm{~min}$ at $4^{\circ} \mathrm{C}$ and collected. The protein concentration of cells in each group was detected by Bradford method to ensure the same amount of protein samples loaded. Then SDS-PAGE was performed, and the cells were transferred to a PVDF membrane. Then, the membrane was sealed with $5 \%$ defatted milk powder solution for $1 \mathrm{~h}$, and incubated with c-myc primary anti-antibody $(1: 1,000)$ and internal reference GAPDH primary anti-antibody $(1: 1,000)$ overnight at $4^{\circ} \mathrm{C}$ and then the HRP labeled secondary antibody for $1 \mathrm{~h}$. Finally, DAB color development solution was applied, and the band optical density value was analyzed.

Statistical analysis. SPSS 17.0 software was employed for analysis. The data were expressed by mean standard \pm deviation, and the mean of two samples was tested by t-test. $\mathrm{P}<0.05$ indicates that the difference is statistically significant.

\section{Results}

Decreased level of miR-125a-3p in peripheral blood of AL children. The qRT-PCR results are exhibited in Fig. 1.
Compared with that in the healthy children group, the miR-125a-3p level in the peripheral blood cells of AL children was remarkably reduced $(\mathrm{P}<0.05)$.

Culture of childhood AL cells. Morphology of cells was observed under a microscope (Fig. 2). Childhood AL cells were seen as small round bright spots, clusters of cells were observed, and there were a great number of cells. Compared with the childhood AL group, cells grew in suspension in the culture plate, with weak opacity and the occurrence of dead cell fragments in the GEM group. In the GEM + miR-125a-3p mimic group, compared with the former two groups, the number of cells was distinctly reduced, the cell state was relatively poor, and more dead cells floated in the culture well.

GEM inhibits the proliferation of childhood AL cells. Fig. 3A presents EdU staining results. Compared with that in the control group, the proliferation rate of cells in the GEM group was alleviated $(\mathrm{P}<0.05)$. Compared with that in the GEM group, the proliferation rate of cells in the GEM + miR-125a-3p mimic group was enhanced $(\mathrm{P}<0.05)$ (Fig. 3B).

GEM promotes apoptosis of childhood AL cells. TUNEL staining results are presented in Fig. 4A. Compared with that in the control group, the apoptosis rate of cells in the GEM group was decreased $(\mathrm{P}<0.05)$. Compared with that in the GEM group, the apoptosis rate of cells in the GEM + miR-125a-3p mimic group increased $(\mathrm{P}<0.05)$ (Fig. 4B). 
A

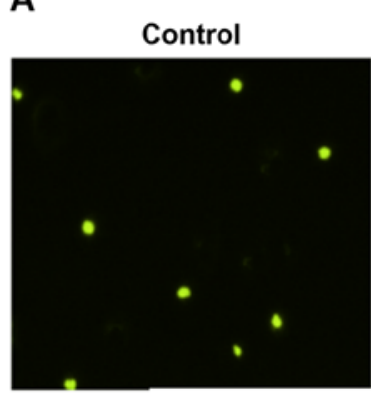

Gemcitabine

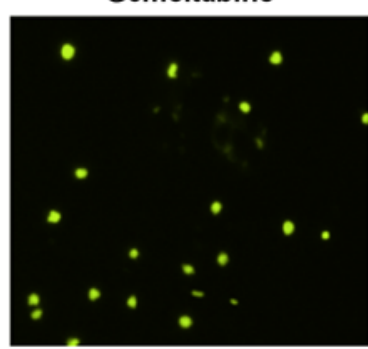

Gemcitabine+ miR-125a-3p mimic

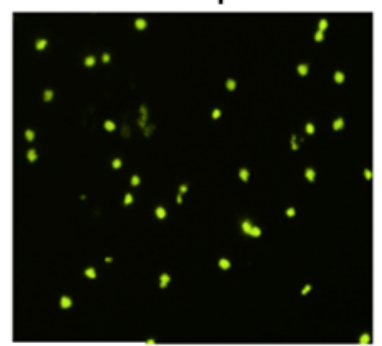

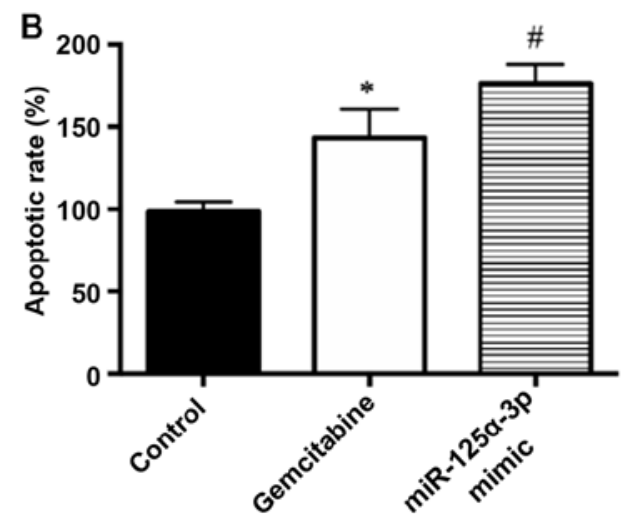

Figure 4. Apoptosis of cells in each group determined through TUNEL assay. (A) TUNEL staining (x20). (B) Statistical graph of apoptosis rate. "P $<0.05$, GEM group vs. control group; ${ }^{\#} \mathrm{P}<0.05$, GEM + miR-125a-3p mimic group vs. GEM group. GEM, gemcitabine; TUNEL, terminal deoxynucleotidyl transferase dUTP nick end labeling.

A

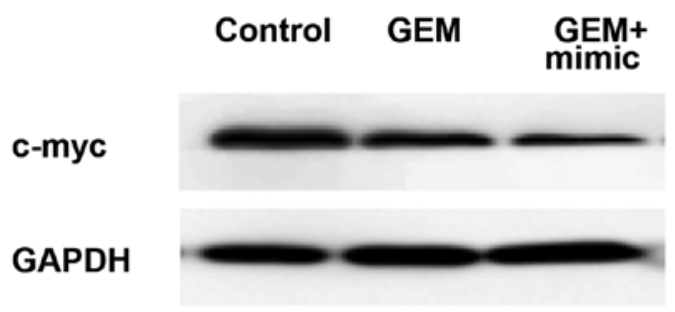

B

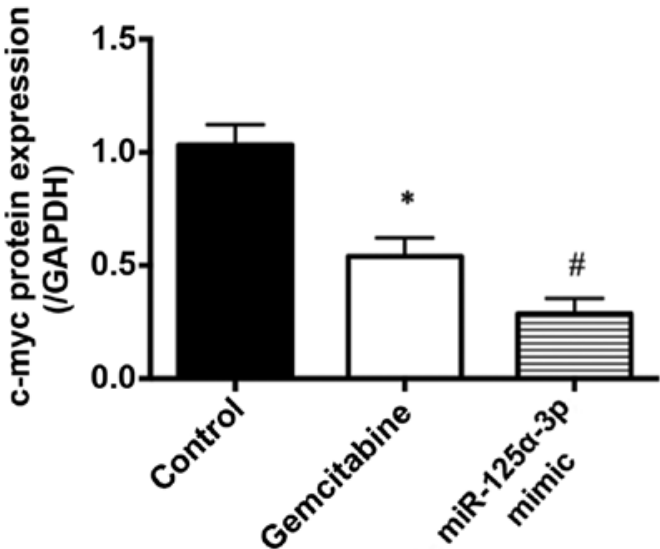

Figure 5. Expression of c-myc protein in each group of cells. (A) Western blotting bands. (B) Statistical graph of bands. "P<0.05, GEM group vs. control group; ${ }^{\text {"}} \mathrm{P}<0.05, \mathrm{GEM}+\mathrm{miR}-125 \mathrm{a}-3 \mathrm{p}$ mimic group vs. GEM group. GEM, gemcitabine.

GEM impedes the protein level of c-myc in childhood AL cells. Western blotting bands are revealed in Fig. 5A. Compared with that in the control group, the protein level of c-myc in the GEM group was downregulated $(\mathrm{P}<0.05)$. Compared with that in the GEM group, the protein level of c-myc in the $\mathrm{GEM}+\mathrm{miR}-125 \mathrm{a}-3 \mathrm{p}$ mimic group was distinctly reduced $(\mathrm{P}<0.05)$, as shown in Fig. 5B.

\section{Discussion}

Childhood AL generally refers to childhood acute lymphoblastic leukemia. Due to abnormal clonal proliferation of precursor B, T or mature B lymphocytes, extensive infiltration of organs and tissues throughout the body destroys normal structures and functions of organs, eventually leading to organ failure and even death $(11,12)$. With the advancement of molecular biology technology, certain progress has been made in the treatment of childhood AL. According to statistics, the 5-year disease-free survival rate of patients with childhood AL can reach $>80 \%$, and some of them can even be cured $(13,14)$. However, there are still patients who can not be effectively relieved even if they are treated. As a result, it is particularly vital to find new anti-leukemia drugs with low toxicity $(15,16)$.
In recent years, research on non-coding RNAs has received increased attention, and the biological function of miRNAs in the body is worth exploring. The generation process of miRNAs in organisms is as follows. The precursor miRNAs are sheared and processed by some nucleases to generate mature miRNAs. They then bind to the target gene mRNA through base complementary pairing to enhance its degradation or hinder its translation into protein (17). Increasingly more studies have proved that miRNAs play a key role in childhood AL. A study discovered the abnormal expression of miRNAs in blood samples of children with AL, and further indicated that miR-125b and miR-203 are potential molecular markers for diagnosing childhood AL (18). In addition, the study by Swellam et al (19) denoted that the levels of miR-143 and miR-182 in bone marrow samples of children with AL are decreased, and the prognosis of patients is poor. It reveals that abnormally expressed miRNAs play a key role in the development and progression of childhood AL.

In this study, the level of miR-125a-3p in the peripheral blood of healthy children and AL children was analyzed. qRT-PCR results showed that the level of miR-125a-3p in the peripheral blood of AL children was reduced as remarkably as $\sim 50 \%$, as shown in Fig. 1 . Then the bone marrow of AL children was collected, and leukemic cells were extracted and separated. GEM was chosen to incubate the cells, and then 
miR-125a-3p mimic was transfected into leukocytes by means of Lipofectamine 2000 transfection technology to explore regulatory effect of GEM on proliferation and apoptosis of childhood AL cells. Subsequently, EdU staining was adopted to investigate the effect of GEM on proliferation of childhood AL cells. The results corroborate that GEM can notably inhibit cell proliferation compared to the control group. TUNEL staining was applied to determine the regulatory effect of GEM on apoptosis of childhood AL cells. The results indicate that GEM can markedly impede apoptosis of cells, and the combined use of GEM and miR-125a-3p mimic has a greater effect on inhibiting apoptosis of cells. miR-125a-3p can bind to its downstream target gene c-myc, which is an important member of oncogenic genes and participates in the regulation of the occurrence and development of various tumors (20). In order to explore the mechanism of GEM, western blotting was chosen to uncover how GEM regulates c-myc, the downstream target of miR-125a-3p. Results are shown in Fig. 5, the protein expression level of c-myc in childhood AL cells was reduced markedly after GEM treatment.

In conclusion, GEM can effectively inhibit proliferation and promotes apoptosis of childhood AL cells. Its mechanism of action may be related to the upregulation of miR-125a-3p to inhibit the expression of c-myc. The results of this study provide an experimental basis for miR-125a-3p to be applied in the treatment of childhood AL.

\section{Acknowledgements}

Not applicable.

\section{Funding}

No funding was received.

\section{Availability of data and materials}

The datasets used and/or analyzed during the current study are available from the corresponding author on reasonable request.

\section{Authors' contributions}

WX wrote the manuscript. WX and YY collected and analyzed general data of patients. SY performed PCR. GL and WX helped with EdU staining and TUNEL staining. All authors read and approved the final manuscript.

\section{Ethics approval and consent to participate}

The study was approved by the Ethics Committee of Yidu Central Hospital of Weifang (Weifang, China) and informed consents were signed by the patients' guardians.

\section{Patient consent for publication}

Not applicable.

\section{Competing interests}

The authors declare that they have no competing interests.

\section{References}

1. Intusoma $\mathrm{U}$, Nakorn $\mathrm{CN}$ and Chotsampancharoen $\mathrm{T}$ : Intracranial hemorrhage in childhood acute leukemia: Incidence, characteristics, and contributing factors. Pediatr Neurol 99: 23-30, 2019.

2. Park SH, Lee MJ, Park CJ, Jang S, Seo EJ, Im HJ and Seo JJ: Bone marrow recovery of hematopoietic stem cells and microenvironment after chemotherapy in childhood acute lymphoblastic leukemia: Consecutive observations according to chemotherapy schedule. Pediatr Hematol Oncol 36: 222-235, 2019.

3. Rahmani M, Fardi M, Farshdousti Hagh M, Hosseinpour Feizi AA, Talebi M and Solali S: An investigation of methylation pattern changes in the IKZF1 promoter in patients with childhood B-cell acute lymphoblastic leukemia. Blood Res 54: 144-148, 2019.

4. Boulet-Craig A, Robaey P, Barlaam F, Laniel J, Oswald V, Jerbi K, Sultan S, Affret-Bertout L, Drouin S, Krajinovic M, et al: Visual short-term memory activation patterns in adult survivors of childhood acute lymphoblastic leukemia. Cancer 125: 3639-3648, 2019.

5. Hara H, Kawamoto T, Fukase N, Kawakami Y, Takemori T, Fujiwara S, Kitayama K, Nishida K, Kuroda R and Akisue T: Gemcitabine and docetaxel combination chemotherapy for advanced bone and soft tissue sarcomas: Protocol for an open-label, non-randomised, Phase 2 study. BMC Cancer 19: 725, 2019.

6. Cornacchia MA, Coromilas AJ, Gallitano SM, Frank RC and Halasz CL: Subacute cutaneous lupus erythematosus induced by gemcitabine in 2 patients with pancreatic cancer. JAAD Case Rep 5: 596-601, 2019.

7. Macchini M, Chiaravalli M, Zanon S, Peretti U, Mazza E, Gianni L and Reni M: Chemotherapy in elderly patients with pancreatic cancer: Efficacy, feasibility and future perspectives. Cancer Treat Rev 72: 1-6, 2019.

8. Mohr AM and Mott JL: Overview of microRNA biology. Semin Liver Dis 35: 3-11, 2015.

9. Simonson B and Das S: MicroRNA therapeutics: The next magic bullet? Mini Rev Med Chem 15: 467-474, 2015.

10. Pepin G and Gantier MP: microRNA Decay: Refining microRNA regulatory activity. MicroRNA 5: 167-174, 2016.

11. Kanderova V, Kuzilkova D, Stuchly J, Vaskova M, Brdicka T, Fiser K,Hrusak O,Lund-Johansen F and Kalina T: High-resolution antibody array analysis of childhood acute leukemia cells. Mol Cell Proteomics 15: 1246-1261, 2016.

12. Madhusoodhan PP, Carroll WL and Bhatla T: Progress and prospects in pediatric leukemia. Curr Probl Pediatr Adolesc Health Care 46: 229-241, 2016.

13. Wiemels J: Perspectives on the causes of childhood leukemia Chem Biol Interact 196: 59-67, 2012.

14. Pui CH, Carroll WL, Meshinchi S and Arceci RJ: Biology, risk stratification, and therapy of pediatric acute leukemias: An update. J Clin Oncol 29: 551-565, 2011.

15. Zimmermannova O, Zaliova M, Moorman AV, Al-Shehhi H, Fronkova E, Zemanova Z, Kalina T, Vora A, Stary J, Trka J, et al: Acute lymphoblastic leukemia with aleukemic prodrome: Preleukemic dynamics and possible mechanisms of immunosurveillance. Haematologica 102: e225-e228, 2017.

16. Pålsson A, Malmström M and Follin C: Childhood leukaemia survivors' experiences of long-term follow-ups in an endocrine clinic - A focus-group study. Eur J Oncol Nurs 26: 19-26, 2017.

17. Umerez M, Garcia-Obregon S, Martin-Guerrero I, Astigarraga I, Gutierrez-Camino A and Garcia-Orad A: Role of miRNAs in treatment response and toxicity of childhood acute lymphoblastic leukemia. Pharmacogenomics 19: 361-373, 2018.

18. Piatopoulou D, Avgeris M,Drakaki I, Marmarinos A, Xagorari M, Baka M, Pourtsidis A, Kossiva L, Gourgiotis D and Scorilas A: Clinical utility of miR-143/miR-182 levels in prognosis and risk stratification specificity of BFM-treated childhood acute lymphoblastic leukemia. Ann Hematol 97: 1169-1182, 2018.

19. Swellam M, Hashim M, Mahmoud MS, Ramadan A and Hassan NM: Aberrant expression of some circulating miRNAs in childhood acute lymphoblastic leukemia. Biochem Genet 56: 283-294, 2018

20. Jackstadt R and Hermeking H: MicroRNAs as regulators and mediators of c-MYC function. Biochim Biophys Acta 1849: 544-553, 2015. 